\title{
How to form the leading innovation capability a multi-case study based on Haier and Huawei
}

\author{
Kaixuan Lou ${ }^{1, a}$, Haibing Liu $^{2, b *}$ \\ ${ }^{1}$ School of Economics and Management, Lanzhou Jiaotong University, Lanzhou, China \\ ${ }^{2}$ School of Economics and Management, Lanzhou Jiaotong University, Lanzhou, China
}

\begin{abstract}
This paper analyzes the innovation practices of Haier Group and Huawei Company since the leading innovation. On the basis of the existing research on "innovation leading", this paper makes full use of the theoretical knowledge of original innovation and meaningful innovation, and tries to explore the formation path of leading innovation ability. In this paper, it is found that: First, the accumulation of original innovation ability suports the formation and the development of leading innovation ability. In the formation and the development of leading innovation ability, it is always accompanied by the accumulation and improvement of the original innovation ability. Second, the realization of leading innovation is always accompanied by the independent and controllable open innovation system. Under the condition of independent and controllable ability, the integration of domestic and foreign high-quality innovation resources for open innovation also plays a certain role in promoting the formation of leading innovation ability. Third, stimulating employees' innovation behavior can stimulate the enthusiasm and creativity of innovation of all employees, and reserve innovation power for enterprises' leading innovation, which is an important factor to promote the formation of enterprises' leading innovation ability. Fourth, the sense of social responsibility conducive to innovation, driven by the entrepreneurial spirit, has formed a culture of innovation among all employees of the enterprise. Innovation and reform have become deeply embedded in the development of the enterprise, providing a value orientation for the formation of the leading innovation ability of the enterprise.
\end{abstract}

\section{Introduction}

The fourth industrial revolution, driven by new technologies such as artificial intelligence and big data, has had a great impact on China's industry, especially the manufacturing industry, and the industrial pattern of the manufacturing industry is changing. If Chinese enterprises want to seize the middle and high end of the manufacturing value chain in the fierce international competition and win the future competitive advantage, they must change the past strategy-oriented strategic guidance and turn to innovation-oriented innovation guidance. Under the background of highly developed information technology and network technology and vigorous development of knowledge economy, China's manufacturing enterprises need to keep up with the pace of The Times, pay more attention to the role of innovation, and lead the development of enterprises with innovation. Therefore, the formation path of leading innovation ability is what enterprises need to explore, but there is hardly any theoretical literature on "leading innovation". Therefore, this paper attempts to explore the formation path of leading innovation ability by mining and analyzing two representative enterprises in the industry.

\section{Theory}

\subsection{Original Innovation}

The research on original innovation began in 1999, when Goldenberg and Mazursky put forward the concept of "Original Innovation". The concept of original innovation is not clearly defined, and researchers in different research fields have different focuses. Among them, Chen Jin summed up two representative points[1] of view are as follows: (1) original innovation has novelty, which is embodied in making important discoveries or inventions that did not exist before for the first time; (2) the results of the original innovation also appear for the first time and are groundbreaking.

To sum up, original innovation means to put forward an unprecedented theory or invent a brand new technology. It is the most powerful and far-reaching independent innovation mode with the capacity of "creative destruction" mentioned by Schumpeter. It has the core significance for the improvement of

a1457091216@qq.com * Corresponding author: b habliu@zju.edu.cn 
productivity and social progress, and is the core embodiment of enterprise creativity. Therefore, the success of original innovation[2] can effectively promote the leapfrog development of science and technology in China, and promote the transformation of enterprises to leading innovation.

\subsection{Meaningful Innovation}

With the development and progress of social culture and the improvement of people's ideas, more and more enterprises begin to pay attention to sustainable development. Most of the traditional innovation paradigm puts the economic benefits of enterprises in the first place, mainly focuses on the internal efficiency and market orientation of enterprises, ignores the cognition of the original meaning of "innovation" and the sustainable development of enterprises, and lacks the sense of social responsibility and reflection on the humanistic spirit.

As a new management paradigm of innovation, sense of innovation(Chen Jin,2018)[3] paradigm shift in the past, driven by market, technology, and will focus on self and social sustainable development, its main purpose is to the enterprise from the bondage of the focus on short-term interests and internal efficiency, gradually to focus on long-term benefits and the external social welfare, the realization of social progress and human development significance of innovation practice.

\subsection{Leading Innovation}

Since the fourth industrial revolution, $\mathrm{Xi}$ Jinping emphasizing the importance of innovation at important conference, and put forward that "innovation is the primary driving force for development", it can be seen that innovation plays an increasingly important role in Chinese enterprises, and "innovation leading" will be the general trend. In order to let more and more enterprises realize the importance of innovation, our country scholar Liu Haibing and Xu Qingrui in 2020 proposed the leading innovation[4], thought innovation is a key factor in enterprise development progress, request with stronger sense of social responsibility as the guide, "guiding and leading" in the form of innovation enterprise strategic choice and implementation, so as to promote enterprise realize benefit value and social value of the benign interaction, promoting the human society progress.

To sum up, original innovation and meaningful innovation are the foundation for the formation of leading innovation. Original innovation is always accompanied by the formation and development of leading innovation ability, and the continuous improvement of original innovation ability and meaningful innovation ability promotes the development of leading innovation ability. Leading innovation is committed to the balance between the interests of enterprises and the interests of society. It can be said that it plays an important role in the future development of Chinese enterprises. Therefore, it is necessary to discuss the path of leading innovation ability. However, as a new innovation paradigm, the formation path of leading innovation ability has not been constructed yet, which is exactly the problem of this paper.

\section{Research methods and data sources}

Case study[5] is to extract undiscovered new conclusions from a phenomenon by using qualitative research method on the basis of abundant case data, so as to reveal the essence of the phenomenon and the law of the development of things. Compared with single case study, multiple case study forms a kind of repetitive logic of case experiment to support or refute research inference through data comparison. Based on the above considerations, this study selects multiple case studies.

\subsection{The Basis Of Case Selection}

Based on the extreme typicality of case selection, this paper adheres to the theoretical sampling and differential logic principle of multi-case study, and attempts to explore the formation mechanism of leading innovation ability by deeply digging and analyzing the case enterprises and extracting the key information. Therefore, the case should have the following characteristics: (1) the case enterprise occupies a leading position in the industry, and is typical and representative; (2) leading innovation plays a key role in improving the core competence of the case enterprise; (3) the enterprise has formed the leading innovation ability, and the forming path of the leading innovation ability is traceable; (4) the case enterprise has abundant available information and data.

Based on the above standards, this paper selects Hair Group (here in after referred to as "Haier") and Shenzhen Huawei Technology Company (here in after referred to as "Huawei") as the sample enterprise in this paper. The main reasons are as follows: (1)Haier and Huawei both take the leading position in their respective industries and play a certain role as role models; (2)Haier and Huawei both adhere to innovation as the core, and have formed leading innovation, which plays a key role in improving the core competence of the enterprise; (3)The innovation management research team where the author works has maintained a cooperative relationship with Haier for 30 years, and has also kept a long-term tracking investigation on Huawei, accumulating a large amount of first-hand information to ensure the reliability and validity of the research.

\subsection{Data Sources}

In order to ensure the reliability and validity of the research, this study collects data from the following two aspects.

(1) Zhejiang University Innovation Management Team has accumulated a large number of first-hand data in the cooperation with Haier and Huawei, forming a wealth of research results. On the basis of combing the 
internal research results, this paper combines a large number of second-hand data to carry out the research. (2)The second-hand materials mainly come from the internal and external materials of the enterprise: the contents related to this study were searched through CNKI database and Wipp periodical database, and the relevant literature on the innovation process and innovation ability of Haier and Huawei were read and the valuable information was mined, and the relevant information on innovation leadership and innovation ability was searched from the published books of Haier and Huawei.

\section{Case analysis}

\subsection{Haier's Leading Innovation}

Haier's transformation from innovation-driven to innovation-led innovation paradigm is a continuous process. In the stages of its brand strategy, diversification strategy, internationalization strategy and globalization strategy, Haier basically relies on strategyoriented innovation to drive, and the innovation leadership is not obvious. After take part in the network, in order to solve the problem of big enterprise disease and make the enterprise obtain sustainable development, Haier based on open innovation system and management mode of "Integration of People and Orders" actively attempt to transform the traditional home appliance industry, is preparing to build a for a better life, in order to meet user needs with way of life as the core of innovation ecosystem. Haier is committed to create a sustainable ecological brand, began to explore innovative leading high quality development path.

With the deepening of innovation degree, Haier has been developing and making progress in such fierce international competition. Among them, the leading innovation plays a key role in promoting Haier's core competence in the specific development process. In the 2018 China brand value list, Haier brand value with 2092.08 billion won the first place in the 2018 (24th) China brand value 100 list. Haier has transformed from a giant of household appliances to an ecological brand of the Internet of things, which marks the initial formation of Haier's leading innovation.

\subsection{Huawei's Leading Innovation}

In the more than 30 years since its establishment, Huawei has been praised and imitated by other enterprises in the industry for attaching great importance to research and development, and has always insisted on a large amount of investment in innovation and research and development. In 2006, the proportion of research and development personnel in the enterprise reached $48 \%$, and it has made a lot of innovative achievements, and the innovation ability of the enterprise has been constantly improved. In 2011, Huawei began to enter the leading innovation stage, and its emphasis on research and development continued to increase, and it set up the "2012 Laboratory". In the stage of global expansion,
Huawei began to conduct research and development of 4G (LTE) technology. After entering the innovation leading stage, Huawei's 4G (LTE) technology has reached the world's leading level, indicating that Huawei starts to explore the leading innovation ability. After 4G (LTE) technology, in order to expand the market and attract more users, Huawei took the lead in the research and development of 5G technology after it predicted and judged the market demand based on its own technical ability. While actively expanding excellent external innovation channels, Huawei integrates global innovation resources, absorbs a variety of heterogeneous knowledge, and is committed to building a fully connected world ecosystem of connectivity, building a new ecology of global communication innovation network, and leading cutting-edge technologies.

Based on its profound technical foundation and advanced research and development capability, Huawei insists on leading the enterprise's development with innovation, makes full use of external excellent innovation resources, and has made great progress in the communication industry. In 2013, Huawei surpassed Ericsson in sales revenue to become the largest telecom equipment manufacturer by sales revenue. In 2019, Huawei was granted a $5 \mathrm{G}$ commercial license by the ministry of industry and information technology of China, with the largest number of commercial contracts for $5 \mathrm{G}$ in the world, making it a communications giant leading the way in $5 \mathrm{G}$ technology. Under the guidance of innovation, Huawei has been promoted from a leading enterprise to a leading enterprise in the field of communication equipment industry, and its leading innovation ability has basically taken shape.

\section{Results and discussion}

\subsection{The formation and accumulation of original innovation capability}

The formation of original innovation ability is a process of dynamic accumulation, which refers to the use of external innovation resources on the basis of making full use of internal resources, so as to achieve the joint function of internal and external resources and make enterprises make significant theoretical or technological discoveries[6]. It is a process of absorbing and internalizing core technology sources. With the continuous investment of enterprises in the field of basic research, their original innovation ability gradually accumulates, and they begin to fulfill more and more social responsibilities to lead the development of the industry, and their leading innovation ability gradually takes shape.

By combing and summarizing the accumulation process of the original innovation ability of the two case companies, it can be concluded that the leading innovation ability of Haier and Huawei is formed in the long-term accumulation process of the original innovation ability. The accumulation of leading innovation ability and original innovation ability is interactive and hard to be separated. Without the 
capacity for original innovation in technology, no matter how innovative the business model, it will not be enough to lead the industry in the search for meaningful innovation across corporate boundaries. Therefore, the accumulation of original innovation ability is the foundation for enterprises to move towards the leading innovation stage. The basic function of original innovation ability is mainly reflected in the following aspects: First, the improvement of original innovation ability provides the necessary expression context for the leading innovation ability. In the process of the accumulation of original innovation ability, the enterprise gradually reaches the middle and high end of the industry value chain and is about to enter the "No Man's Land" of market competition. At this time, the enterprise urgently needs to realize disruptive innovation by leading the innovation ability and move to a higher development stage. Second, original innovation is an important support for the formation of leading innovation ability. Leading innovation ability requires enterprises to master enough basic knowledge while continuously absorbing heterogeneous knowledge[7], grasp the evolution direction of technology, and simulate the forming atmosphere of technology window. In the logic of disruptive innovation, the order of existing resources is disrupted and rearranged, increases the possibility of knowledge collision, and constructs the forming atmosphere of subversive innovation. The technological control provided by the original innovation ability provides a strong guarantee for enterprises to deal with the window of opportunity under the innovation leading mechanism.

\subsection{Independent and controllable open innovation system}

Independent and controllable open innovation is a necessary condition for enterprises to realize innovation guidance. By building an independent and controllable open innovation system, enterprises can form leading innovation capability on the basis of absorbing and digesting external high-quality resources, so as to lead the development of the industry.

Haier and Huawei have always been accompanied by open innovation on the road from innovation-driven to innovation-led. In the process of open innovation, Haier to avoid repeating the Boeing, Procter \& Gamble, etc, on the basis of reference to external resources with high quality and pay attention to the accumulation of knowledge and learning itself, so that the open innovation on the basis of independent continues to enhance its capability of controllable, and by building its own open innovation system to promote innovation driven by innovation to lead the cross. Huawei has started open innovation since about 1997. It pays attention to the improvement of its own research and development ability and technical ability, and always insists on the utilization of external resources, which also promotes the accumulation of Huawei's innovation ability and lays a foundation for the realization of leading innovation. Such as the establishment of the
"2012 Laboratory," focus on the future research and development, it also means that Huawei at this time of open innovation is not only for integration of external resources, but more important to research the allocation of resources in the field of innovation for future demand. Therefore, this stage can be called the independent and controllable open innovation stage. On the basis of adhering to the utilization of external resources, Huawei pays attention to the accumulation of its own knowledge, which promotes the accumulation of Huawei's innovation ability and lays a foundation for the realization of leading innovation.

To sum up, the enterprise through the establishment of autonomous control system of open innovation, on the basis of the use of external quality resources, through digesting and absorbing useful knowledge for enterprise development, and internalize the knowledge of the enterprise itself, and thus enhance technical research and development ability, makes the enterprise in the capacity for independent controllable growing under the condition of continuous innovation, to create an innovation ecosystem, gradually formed lead innovation ability.

\subsection{Incentives for employee innovation}

The incentive of the enterprise to the innovation behavior of the employees stimulates the innovation enthusiasm and potential of the employees, and makes all the employees contribute their wisdom and participate in the innovation actively, which plays an important role in the formation of the leading innovation ability. It can be said that the incentive of employees' innovation behavior is one of the key paths for the formation of enterprises' leading innovation ability.

In the development process of Haier and Huawei, they always adhere to the people-oriented, pay attention to the incentive of employees' innovative behavior, and pay attention to the cultivation of innovative talents. In order to stimulate the innovation of employees, Haier put forward such incentive behaviors as "integration of people and orders" and "everyone is the CEO" and named products or accessories after the employees, so as to stimulate the innovation enthusiasm of employees and promote the improvement of the innovation ability of the enterprise. Huawei as an employee-owned privately held company, in the process of incentive for innovation behavior of the employees, in addition to the material incentive and spiritual incentive, and return on equity incentive, let employees holding the enterprise equity within the fixed phase, by participating in enterprise profit sharing to get value-added benefits, achieve longterm incentive for employees, effectively unite staff and the company into a tight community of interests.

From the common experience of Haier and Huawei, the incentive mechanism of employees' innovation behavior is different from the general material incentive, and focuses more on the spiritual value creation. In the formation mechanism of leading innovation ability, the incentive mechanism of employees' innovation behavior has the following core elements: First, give employees the same spiritual value of innovation. Encourage 
employees to actively participate in innovation, so that employees have equal opportunities to participate in the enterprise's innovation, and give employees some spiritual rewards when they excellently complete innovation tasks. Second, give employees the same value for innovation. When rewarding employees, we should not only give them spiritual incentives, but also give them fair material rewards. Finally, give employees innovation-oriented cultural incentives. A strong innovation atmosphere should be formed inside the enterprise, and gradually condensed into the innovation culture of the enterprise, so that employees can actively contribute their own strength in such an environment conducive to innovation. Under the incentive mechanism of employees' innovation behavior, it guides all employees of the enterprise to participate in innovation, and makes the enterprise gradually turn from innovationdriven to innovation-led under the promotion of the innovation force of all employees.

\subsection{Social responsibility drive for innovation}

In the current turbulent international situation, in order to achieve sustainable development and lead the development trend of the whole industry, enterprises must get rid of the constraint of focusing on short-term interests, gradually shift to focus on medium and long term benefits and external social welfare. Driven by the social responsibility that is conducive to innovation, the innovation leadership is realized and the leading innovation ability is formed.

Meaningful innovation (Chen Jin, 2018)[3] emphasizes the real meaning of "innovation" and makes enterprises pay more attention to sustainable development. On the basis of meaningful innovation, guided by social responsibility, leading innovation (Liu Haibing, 2020)[4] is committed to achieving the dual balance between social interests and self-interests, and attaches more importance to realizing self-interests in the process of promoting social progress. Both innovation paradigms emphasize that enterprises must put social interests above their own interests, if they want to achieve sustainable development and develop the ability to lead innovation. Haier and Huawei as the leading enterprises in the industry, actively respond to the call of The Times, and turn their eyes to future social interests and social progress in the context of social responsibility and social interests. Haier has set up many industrial innovation centers to fulfill social responsibilities and realize its own interests on the premise of meeting social needs. Huawei has continuously invested a lot of money in the research and development and design of the underlying architecture of the Chip, Internet, Huawei Cloud and other objects, aiming to get rid of the dependence on foreign technology, better fulfill its social responsibilities while developing itself, and realize the sustainable development of the enterprise.

To sum up, the sense of social responsibility conducive to innovation, driven by the entrepreneurial spirit, has formed a culture of innovation among all employees, and innovation and reform have become deeply embedded in the development of enterprises. Haier and Huawei as the leading enterprises in the industry, are leading the development of surrounding industries in the process of fulfilling their social responsibilities, forming an industrial innovation ecology, and leading the innovation driven by the social responsibility conducive to innovation.

\section{Conclusions}

By analyzing the innovation practices of Haier and Huawei, this paper tries to answer the question of "how to form the leading innovation ability" on the basis of fully drawing on the theoretical knowledge of original innovation and leading innovation.

(1) The formation and accumulation of original innovation ability supports the formation and development of leading innovation ability. With its novelty and breakthrough characteristics, original innovation has accumulated a large number of innovation achievements for enterprises and promoted the formation of enterprises' leading innovation ability. It can also be said that the formation of the leading innovation ability is always accompanied by the accumulation of the original innovation ability.

(2) Promote the leading innovation process of enterprises by building an independent and controllable open innovation system. The independent and controllable open innovation mode, systematic research and development strategy jointly create the innovation ecology of enterprises, and lay the foundation of innovation ability for leading innovation.

(3) Attaching importance to the cultivation of innovative talents and the encouragement of employees' innovative behaviors, creating a strong innovation atmosphere for the formation of leading innovation, is an important factor to promote the formation of leading innovation ability.

(4) The social responsibility drive for innovation is a key factor for enterprises to move towards leading innovation and form leading innovation ability. It is impossible to form a leading innovation capability to only focus on the enterprise's own interests and ignore the overall interests of the society. Driven by the social responsibility conducive to innovation, enterprises gradually lead the development of surrounding industries to form an industrial innovation ecology.

\section{ACKNOWLEDGMENT}

The authors greatly acknowledge the sponsorship provided by National Nature Science Foundation of China(71572177), Chinese Academy of Engineering (2017-XY-39), Tianyou Youth Talent Lift Program of Lanzhou Jiaotong University. Haibing Liu is correspondence author of this paper. 


\section{References}

1. W. N. Zhou. "Reflections on original innovation and national competitiveness", Beijing, vol. 11, 2007, pp. 53-60.

2. Y. L. Pei, X. Jiang and H. Liu. "Strategic flexibility, original innovation and enterprise competitiveness: the regulatory role of organizational legitimacy", Beijing, vol. 3, 2013, pp. 446-455.

3. J. Chen and G. N.Qu. "Meaningful innovation is an innovative paradigm leading to the revival of philosophy and humanistic spirit in the new era" , Beijing, vol. 7, 2018, pp. 1-9.
4. H.B. Liu and Q. R. Xu,. "From driving to leading: the concept and process of "innovation leading" -- a longitudinal case study based on Haier Group (1984-2019)", Guangxi, vol.1, 2020, pp. 134-149.

5. EISENHARDT K. "Building Theories from Case Study Research.” vol.14, 1989, pp. 532-550.

6. H. Chen and S. X. Gao, "Research on the relationship between dual market learning, original innovation ability and enterprise competitiveness", Beijing, vol.1, 2018.

7. H. B. Liu and Q. R. Xu, "Latecomer firms strategy evolution,innovation paradigm and capabilities evolution," Beijing, vol. 36, 2018, pp. 1442-54. 